\title{
Stiffness-based modelling of a hydraulically-actuated soft robotics manipulator
}

\author{
Lukas Lindenroth*, Junghwan Back, Adrian Schoisengeier, Yohan Noh, \\ Helge Würdemann, Kaspar Althoefer, Hongbin Liu
}

\begin{abstract}
This work investigates the applicability of stiffness-based modelling in soft robotics manipulation. The methodology is introduced and applied to model a soft robotics manipulator as single 3d Timoshenko beam element. The model is then utilized to solve the forward kinematics problem for the manipulator. The algorithm is validated comparing the simulated deflection with the deflection of the physical manipulator for two defined pressure sequences. It is shown that the model behaves in a highly similar fashion in comparison to the manipulator. For both trajectories the maximum position error is close to $6 \mathrm{~mm}$ while the error in orientation not more than $18^{\circ}$. The methodology as described in this work reveals great applicability to the field of soft robots being limited only by the stiffness matrix assembly for the given system. Implementations of inverse kinematics and the effects of external force applications are effectively integrable in the described theory.
\end{abstract}

\section{INTRODUCTION}

Despite large research effort in the field of soft robotics, modelling and accurate controllability are still considered a major challenge in recent investigations [1], [2]. Due to highly individualised soft robotics designs resulting in diverse kinematics descriptions, a universal solution with general applicability has not been found yet. The development of models considering external forces can be difficult to integrate and contradictory with assumptions made. To approach the described issues we examine the implementation of a highly flexible stiffness-based modelling approach which can not only be utilized to solve the forward and inverse kinematics problems based on actuation forces and moments for a given soft robot, but also integrate external forces which are applied to the structure. The model is validated in a forward kinematics algorithm which is applied to a soft robotics manipulator. The latter is developed employing hydraulic actuators, while the modelling itself is similarly applicable to other fluid-based actuation techniques. The tip position and orientation of the robot are evaluated using an implemented optical marker tracking system.

\section{BACKGROUND}

The modelling in soft robots requires the derivation and solution of complex analytical formulations which are often highly individualized to the given robot. In [3] and [4] a mapping technique is derived and investigated to discretise the shape of a soft robot for describing it with

* indicates the corresponding author, email: Lukas.Lindenroth@kcl.ac.uk The authors Lukas Lindenroth, Junghwan Back, Adrian Schoisengeier, Yohan Noh, Helge Würdemann, Kaspar Althoefer and Hongbin Liu are with Department of Informatics, King's College London, UK, WC2R 2LS.

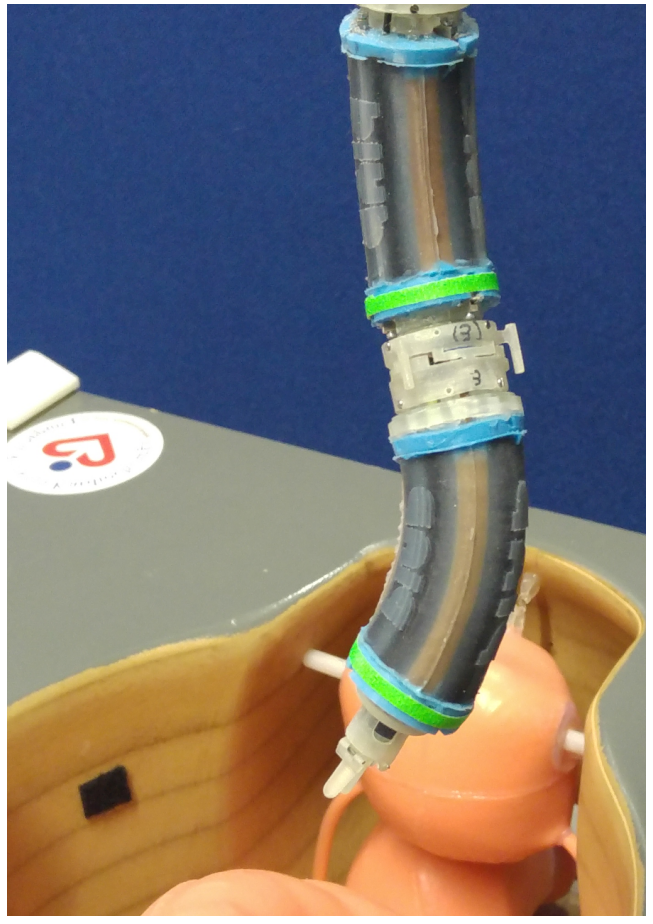

Fig. 1. Serial soft robotics manipulator

the application of classical joints and the commonly used Denavit-Hartenberg method in a similar fashion to hyperredundant robots. It has been found that the model accuracy is then dependent on the number of discretised steps with a computational demand proportional to the latter [5]. In [6] a closed analytical description has been found to derive the kinematics of a hyper-redundant elephant trunk manipulator with applicability to soft robotics. An overview on soft robotics kinematic modelling under the constant curvature constraint in given in [7]. Fast computation is required for kinematics or dynamics models to be efficiently applied in soft robotics control. Therefore a balance between model simplicity and accuracy has to be found. Current state-of-theart suggests that dynamics modelling, although it has been shown be applicable in soft robots [8], is most likely to be too computationally expensive for real-time control [2].

The manipulator as described in [9] is modelled in [10] under the constant curvature assumptions to describe the tip position and orientation based on its arc length and curvature. The model is used to derive forward and inverse kinematics of the manipulator. [11] follows a similar method by assu- 


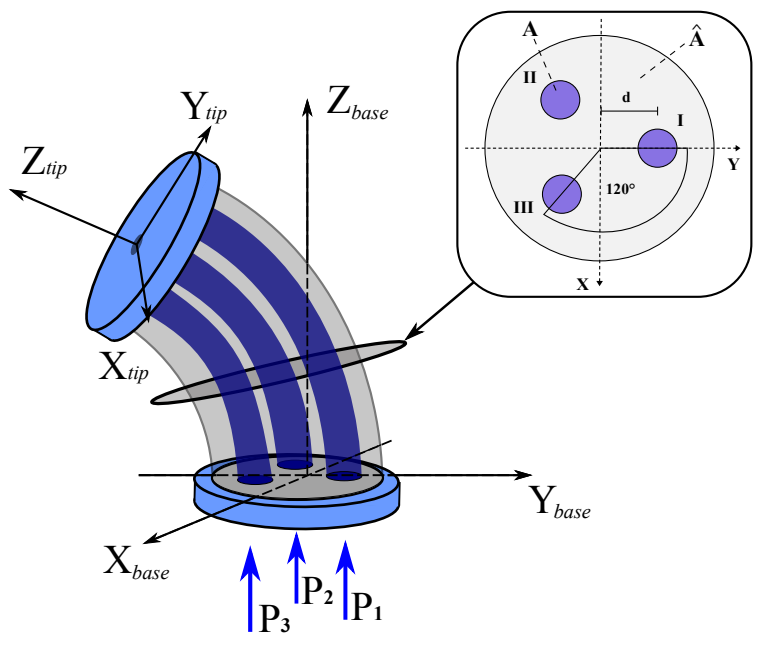

Fig. 2. Soft robotics segment with cross-section

ming piecewise constant curvature. An algorithm is presented defining the segment curvature of the manipulator which is then used to calculate the forward kinematics equations. To solve the inverse kinematics an iterative Jacobian transpose method is employed. The cable-actuated manipulator in [12] follows a similar model assuming virtual joints as previously described in [3] and [4]. A mapping between actuation, virtual joint and task space is then defined and forward as well as inverse kinematics are solved by applying the Denavit-Hartenberg method. In recent work, machine learning is often utilized to find inverse kinematics equations for a soft robot [13] or to validate the manipulator's motion gained from analytical modelling [9]. [14] employ a recurrent neural network to learn the manipulator's dynamics, which shows efficient applicability. Highly diverse approaches to soft manipulator modelling can be found in recent work. While applications are shown in which the constant curvature assumption is employed [9], [11], which does not hold for external force applications. Other work discretises the continuous behaviour of soft robots to approximate it applying virtual joints [12]. Machine learning is an alternative which also shows promising results soft robot modelling. It can be seen that the models derived for describing soft robotics kinematics are individualized and based on a variety of different principles. This work therefore examines a universal approach based on Timoshenko beam theory which could be flexibly utilized for different soft robotics setups.

\section{SYSTEM DESIGN}

A soft robotics manipulator, as presented in figure 2, utilizing hydraulic actuation is developed to validate the modelling approach. The manipulator consists of a silicone rubber module as investigated in prior research [9]. Three inflatable chambers are inserted into the cylindrical body which contain threading to constrain lateral expansion upon pressurization, a cross-section of the manipulator is shown in figure 1. Upon pressurization, the chambers elongate without expanding radially, causing, due to their off-centered

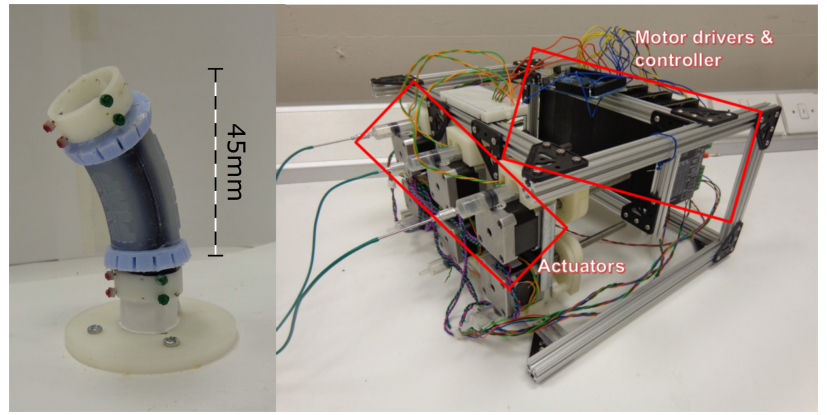

Fig. 3. Bent soft robotics segment with coloured optical markers \& actuators with control and driver units

placement inside the body, bending moments and elongation in the tip. The segment comprises a diameter of $25 \mathrm{~mm}$ and a deflated length of approximately $45 \mathrm{~mm}$.

\section{A. Actuation}

Stepper motor based actuators are developed to provide hydrostatic pressure to the soft robotics structure. The construction comprises a hydraulic syringe which is linked to the fixed motor housing as well as a piston which is attached to a travelling nut on the rotating lead-screw. The constraint in rotation of the piston as well as the overall fixation of both components allow a conversion from rotary screw motion to linear piston displacement. The syringes are designed to provide a maximum hydraulic fluid volume of $1.8 \mathrm{ml}$ and are limited mechanically as well as electronically by embedded contact switches. The stepper motors (Nema 17, Polulu, USA) are driven by stepper motor drivers (2M542N, SainSmart, USA) which are controlled by an Arduino Mega 2560 microcontroller. Individual steps are initiated through a generated PWM signal while the direction of the motor rotation is defined employing a digital output. The contact switches are utilized to drive the motor in an open loop control configuration. Upon system start-up the actuators move towards the contact switches until the latter are engaged to generate accurate initial actuator positions. During the following movements the displacements are tracked on a software level which allows accurate positioning in an open-loop configuration. The actuators are moved with constant velocities. The pressure output as provided by the hydraulic actuators with connected soft robotics segment is measured using diaphragm- and strain-gauge-based pressure transducers (NBP-4, Honeywell, USA) connected through an instrumentation amplifier circuit to analogue inputs of the Arduino microcontroller. The pressure is monitored over the insertion of $1.8 \mathrm{ml}$ into the segment, showing a mostly linear pressure range from $0 \mathrm{kPa}$ to $160 \mathrm{kPa}$ with minor nonlinearities occurring from minor undesired lateral expansion of the inflation chambers. In the following, the pressure output of the actuators is assumed to be in the described linear range.

\section{B. Feedback}

A visual marker tracking system is developed to determine base and tip positions as well as orientations of the segment. 
The segment's base and tip are equipped with connective elements comprising optical markers, which can be seen in figure 3. Two cameras are utilized in a stereoscopic setup comprising perpendicular optical axes to determine the projections of the markers in the $\mathrm{x}-\mathrm{z}-$ and $\mathrm{y}$-z-planes. A computer vision algorithm is employed to find binary image representations of the markers based on their colours, to determine their centre locations in the image based on a Hough circle transform and to track each marker utilizing prior velocity and marker label information. The marker tracking feedback is utilized to determine the tip displacement as projections into the $\mathrm{x}-\mathrm{z}-$ and $\mathrm{y}$-z-planes in the following experimental validation. The marker locations are furthermore used to calculate the tip rotation angles.

\section{STIFFNESS MODELLING}

Linear elastic beam theory describes the deformation of a body under applied loading based on a linear relation between occurring stresses and strains. Different theories have been emerged on this basis, holding for specific assumptions to the properties of the body. The most commonly applied Euler-Bernoulli beam theory assumes a strictly perpendicular orientation between central axis of the beam and its end plate. This, however, neglects shear deformations occurring from applied forces. While this assumption holds for long beams with negligible diameter, shorter beams are not adequately described. This work therefore utilizes Timoshenko beam theory which has been shown to be more suitable for short beams [15]. In beam theory the deflection of a $3 d$ cantilever beam can be related to the applied forces and moments by

$$
\mathbf{T}=\mathbf{K} \cdot \mathbf{X}
$$

With the force and moment vector

$$
\mathbf{T}=[\mathbf{F}, \mathbf{M}]^{T}=\left[F_{x}, F_{y}, F_{z}, M_{x}, M_{y}, M_{z}\right]^{T}
$$

Describing the global forces $F_{i}$ and $M_{i}$ moments acting on the free tip in direction $i$ and

$$
\mathbf{X}=[\boldsymbol{\delta}, \boldsymbol{\theta}]^{T}=\left[\delta_{x}, \delta_{y}, \delta_{z}, \theta_{x}, \theta_{y}, \theta_{z}\right]^{T}
$$

Indicating the displacements in position $\left(\delta_{i}\right)$ and orientation $\left(\theta_{i}\right)$ in $i$. The two vectors are related through the $6 \times 6$ element matrix $\mathrm{K}$, which is known as the stiffness matrix of the beam. In this work we describe the developed soft robotics manipulator as a single $3 \mathrm{~d}$ Timoshenko beam element. Its element stiffness matrix can be defined, as derived in [16], by

$\mathbf{K}=$
$\left[\begin{array}{cccccc}\frac{12 E I}{(1+\phi) L^{3}} & 0 & 0 & 0 & \frac{6 E I}{(1+\phi) L^{2}} & 0 \\ 0 & \frac{12 E I}{(1+\phi) L^{3}} & 0 & -\frac{6 E I}{(1+\phi) L^{2}} & 0 & 0 \\ 0 & 0 & \frac{E A}{L} & 0 & 0 & 0 \\ 0 & -\frac{6 E I}{(1+\phi) L^{2}} & 0 & \frac{(4+\phi) E I}{(1+\phi) L} & 0 & 0 \\ \frac{6 E I}{(1+\phi) L^{2}} & 0 & 0 & 0 & \frac{(4+\phi) E I}{(1+\phi) L} & 0 \\ 0 & 0 & 0 & 0 & 0 & \frac{G J}{L}\end{array}\right]$

With

$$
\phi=\frac{12 E I}{G(\hat{A} / \alpha) L^{2}}
$$

Where $G$ is the shear modulus of the beam, $J$ is the torsion constant and $\alpha$ the Timoshenko coefficient based on the geometry of the beam and $\hat{A}$ the beam's cross section. $E$ refers to the Young's modulus of the beam material, $I$ to it's area moment of inertia and $L$ to it's length. This relation allows the calculation of forces and moments required for a specified deflection of the beam. Matrix $K$ is invertible which leads to

$$
\mathbf{X}=\mathbf{C} \cdot \mathbf{T}
$$

Where $\mathbf{C}=\mathbf{K}^{-1}$ is the analytically defined compliance matrix of the system. The above derived equations offer two possible applications in soft robotics manipulation

- The formulation of kinematics equations of the soft robot based on the applied actuation forces and moments.

- The calculation of deflection occurring from externally applied forces and moments to the soft robot.

In the following, both points are addressed while the first is experimentally validated employing the physical robot.

\section{KINEMATICS MODELLING}

As stated above, a first step in deriving the forward kinematics of the system is to relate the actuation forces and moments to the reference frame corresponding to the stiffness matrix description. In the given case the latter is expressed in regards to the manipulator tip. With the actuation pressures already acting on the tip plane of the manipulator a coordinate transformation is not required. The force acting in chamber $i$ of the soft robotics module is given as

$$
F_{i}=P_{i} \cdot A
$$

Where $P_{i}$ is the pressure on end surface $A$ of chamber $i$. Due to the geometrical location of the inflation chambers the forces are translated to moments about the respective axes. With the given distance of the chambers from the central axis, the corresponding moments can be determined by

$$
M_{i}=d_{i} \times F_{i}
$$

Where $d_{i}$ is the direction vector from the central axis pointing towards force vector $F_{i}$. For the soft robotics segment the chambers are aligned in $7 \mathrm{~mm}$ distance towards the centre with angles of $120^{\circ}$ between one another. The placement of the vertical chambers in the global frame are shown in figure 2. For chamber 1 the position vector in the global reference frame is given as

$$
\boldsymbol{d}_{\mathbf{1}}=\left[x_{1}, y_{1}, z_{1}\right]^{T}=[0 \mathrm{~mm}, 7 \mathrm{~mm}, 0 \mathrm{~mm}]^{T}
$$

The position vectors of chambers 2 and 3 can be obtained by rotating $d_{1}$ about the $\mathrm{z}$-axis using rotation matrices

$$
d_{2}=R_{z, 120^{\circ}} \cdot d_{1}, \quad d_{3}=R_{z, 120^{\circ}} \cdot d_{2}
$$


with the rotation matrix around the global z-axis

$$
\boldsymbol{R}_{\boldsymbol{z}, \mathbf{1 2 0}^{\circ}}=\left[\begin{array}{ccc}
\cos \left(120^{\circ}\right) & -\sin \left(120^{\circ}\right) & 0 \\
\sin \left(120^{\circ}\right) & \cos \left(120^{\circ}\right) & 0 \\
0 & 0 & 1
\end{array}\right]
$$

The forces, pointing along the positive z-axis, are transformed and summed using the principle of superposition

$$
\left[M_{x}, M_{y}, M_{z}\right]^{T}=M_{1}+M_{2}+M_{3}
$$

Which determines the tip moments required for the stiffness matrix calculation in equations 1 and 2 . The mapping from input pressures to global bending moments can be achieved in a compact matrix form as

$$
M_{\text {act }}=\hat{\boldsymbol{U}} \cdot\left[F_{1}, F_{2}, F_{3}\right]^{T}
$$

With the $2 \times 3$ matrix

$$
\hat{\boldsymbol{U}}=\left[\begin{array}{ccc}
d_{1, y} & d_{2, y} & d_{3, y} \\
0 & d_{2, x} & d_{3, x}
\end{array}\right]
$$

Relating the three force vectors to moments using linear algebra. With the achieved mapping the input pressures can now be related to the manipulator's tip deflection through the actuating bending moments $\boldsymbol{M}_{\boldsymbol{a c t}}$ as well as external forces $\boldsymbol{F}_{\text {ext }}$ and moment $\boldsymbol{M}_{\text {ext }}$ can be determined as

$$
\left.\boldsymbol{X}=\boldsymbol{C} \cdot\left[\begin{array}{c}
\boldsymbol{F}_{\text {ext }} \\
0
\end{array}\right]+M_{\text {ext }}\right]
$$

Which can be written as

$$
\left.\left[\begin{array}{l}
\delta \\
\theta
\end{array}\right]=\left[\begin{array}{ll}
C_{11} & C_{12} \\
C_{21} & C_{22}
\end{array}\right] \cdot\left[\begin{array}{c}
F_{\text {ext }} \\
M_{a c t} \\
0
\end{array}\right]+M_{\text {ext }}\right]
$$

Where the displacement $\delta$ and the rotation $\theta$ of the manipulator tip can be formulated as

$$
\begin{gathered}
\delta=C_{11} \cdot F_{e x t}+C_{12} \cdot\left(\left[\begin{array}{c}
M_{a c t} \\
0
\end{array}\right]+M_{e x t}\right) \\
\theta=C_{21} \cdot F_{e x t}+C_{22} \cdot\left(\left[\begin{array}{c}
M_{a c t} \\
0
\end{array}\right]+M_{e x t}\right)
\end{gathered}
$$

Where $\boldsymbol{F}_{\text {ext }}=\left[F_{x}, F_{y}, F_{z}\right]^{T}$ are the externally applied forces, $\boldsymbol{M}_{\text {ext }}=\left[M_{x}, M_{y}, M_{z}\right]^{T}$ the external moments and $M_{a c t}$ the actuation moments as stated above. This relation therefore provides efficiently the possibility to be applied in static force scenarios where external loading is added to the manipulator tip.

Beam theory applied with a single Timoshenko beam element comprises two limitations for the given application:

- The elongation of the segment resulting from applied pressures is not considered due to the formulation as bending moments.

- Deflection along the z-axis is also not defined for occurring bending moments.

In the following sections both limitations are addressed.

\section{A. Elongation compensation}

The elongation of a beam through longitudinal loading can be described as

$$
d L=\frac{L}{E \hat{A}} \cdot F_{e l}
$$

Where $d L$ is the contraction or extension of the beam, $L$ its unloaded length, $\hat{A}$ its cross-section and $F_{e l}$ the elongating force. In the given manipulator it can be approximated that

$$
\boldsymbol{F}_{\boldsymbol{e l}}=A \cdot \frac{P_{1}+P_{2}+P_{3}}{3}
$$

The determined elongation is applied to the deflected tip in form of a translation in the tip direction in an additional iteration step. Based on equation 2 and the analytically found compliance matrix of the system, the rotation of the tip caused by the actuation pressures can be found with

$$
\left[\theta_{x}, \theta_{y}\right]^{T}=\left[\begin{array}{cc}
0 & -\frac{L^{2}}{2 \cdot E I} \\
\frac{L^{2}}{2 \cdot E I} & 0
\end{array}\right] \cdot \hat{\boldsymbol{U}} \cdot A \cdot\left[P_{1}, P_{2}, P_{3}\right]^{T}
$$

The rotations $\theta_{x}$ and $\theta_{y}$ can then be employed to determine the tip direction vector

$$
\boldsymbol{O}_{t i p}=\left(\boldsymbol{R}_{\boldsymbol{x}, \boldsymbol{\theta}_{\boldsymbol{x}}}+\boldsymbol{R}_{\boldsymbol{y}, \boldsymbol{\theta}_{\boldsymbol{y}}}\right) \cdot \boldsymbol{u}_{\boldsymbol{z}}
$$

where $\boldsymbol{O}_{\text {tip }}=\left[O_{t i p, x}, O_{t i p, y}, O_{t i p, z}\right]^{T}$ is the tip orientation vector, $\boldsymbol{u}_{\boldsymbol{z}}$ the unit vector in z-direction and $\boldsymbol{R}_{\boldsymbol{x}, \boldsymbol{\theta}_{\boldsymbol{x}}}$ and $\boldsymbol{R}_{\boldsymbol{y}, \boldsymbol{\theta}_{\boldsymbol{y}}}$ the rotation matrices around $\mathrm{x}$ - and $\mathrm{y}$-axis. The deflected tip with applied elongation is then calculated as

$$
\delta^{+}=\delta+\frac{O_{t i p}}{\left|O_{t i p}\right|} \cdot d L
$$

\section{B. Deflection along the z-axis}

To determine the $\mathrm{z}$-deflection of the beam its curvature $\kappa$ is determined in $\mathrm{X}-\mathrm{z}$ - and $\mathrm{y}-\mathrm{z}$-plane by

$$
\kappa_{i}=\frac{M_{i}}{E I}=\frac{M_{a c t, i}+M_{e x t, i}}{E I} \quad \text { for } i=\{x, y\}
$$

Based on the determined curvatures, the z-coordinate can be calculated as

$$
\boldsymbol{\delta}_{\boldsymbol{z}}^{+}=\frac{1}{\kappa} \cdot \sin (\kappa \cdot(L+d L))+\boldsymbol{\delta}_{\boldsymbol{z}}
$$

Where $\delta_{z}$ is previously defined by the externally applied forces. In the following section the algorithm is validated utilizing the previously described soft robotics manipulator without external loading. The z-deflection is determined based on the curvature in the $\mathrm{x}$-z-plane.

\section{EXPERIMENT}

The forward kinematics algorithm is experimentally validated by calculating the model deflection for two different actuation pressure sequences and applying the latter to the physical manipulator without external loading. Both results are then compared in regards to their displacement in $\mathrm{x}-, \mathrm{y}$ and $\mathrm{z}$-axis as well as in their rotation about $\mathrm{x}$ - and $\mathrm{y}$-axis. The first motion, as shown in figure 4(a) comprises an inflation of chamber one from $0 \mathrm{kPa}$ to $160 \mathrm{kPa}$ followed by a deflation from $160 \mathrm{kPa}$ to $0 \mathrm{kPa}$ while maintaining a constant pressure in both of the unused chambers. The second trajectory, 

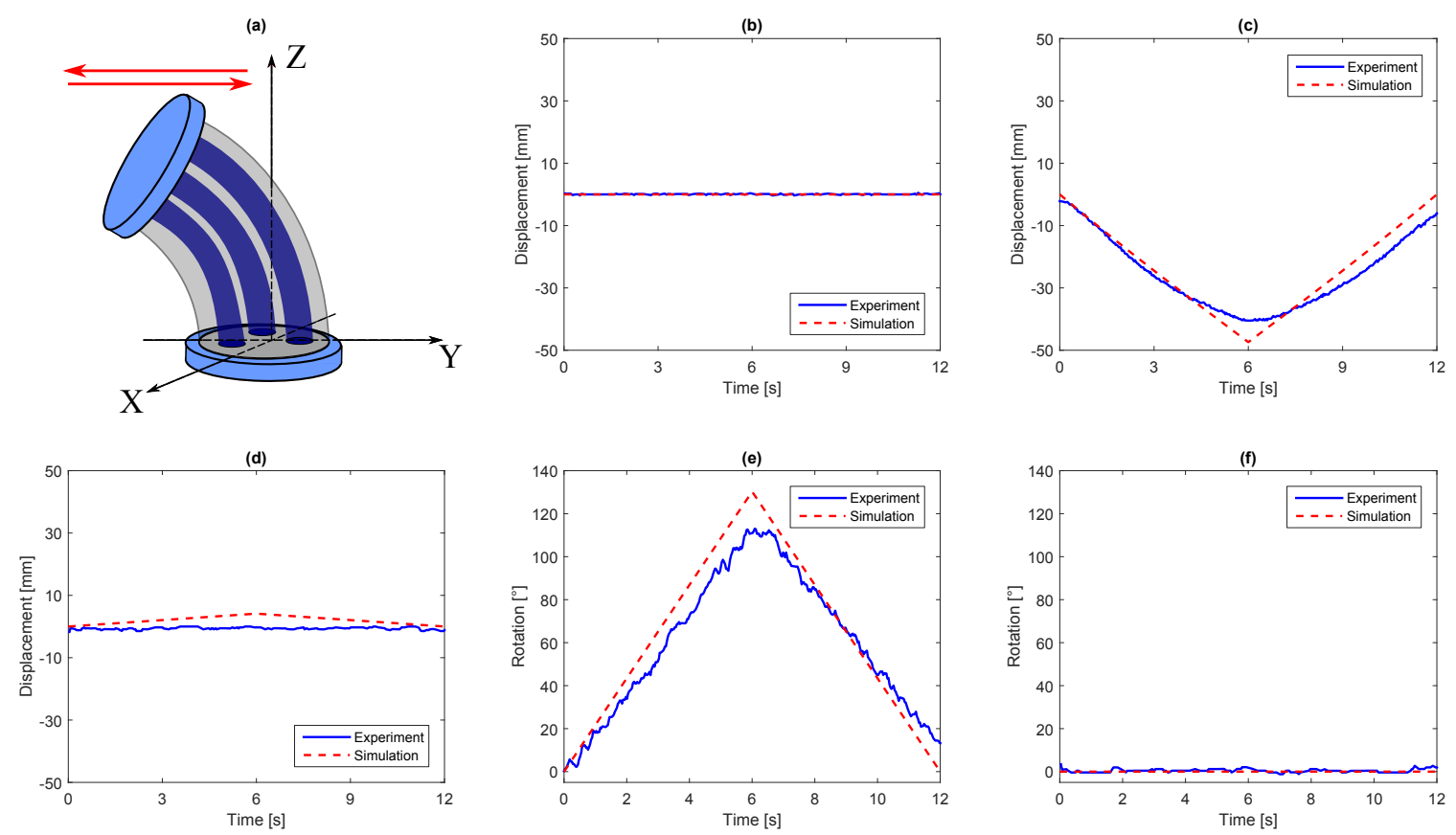

Fig. 4. Experimental results for first trajectory with (a) Trajectory visualization, deflection in $x-(b)$, y- (c) and z-axis (d) as well as rotation around $x-$ (e) and y-axis (f)
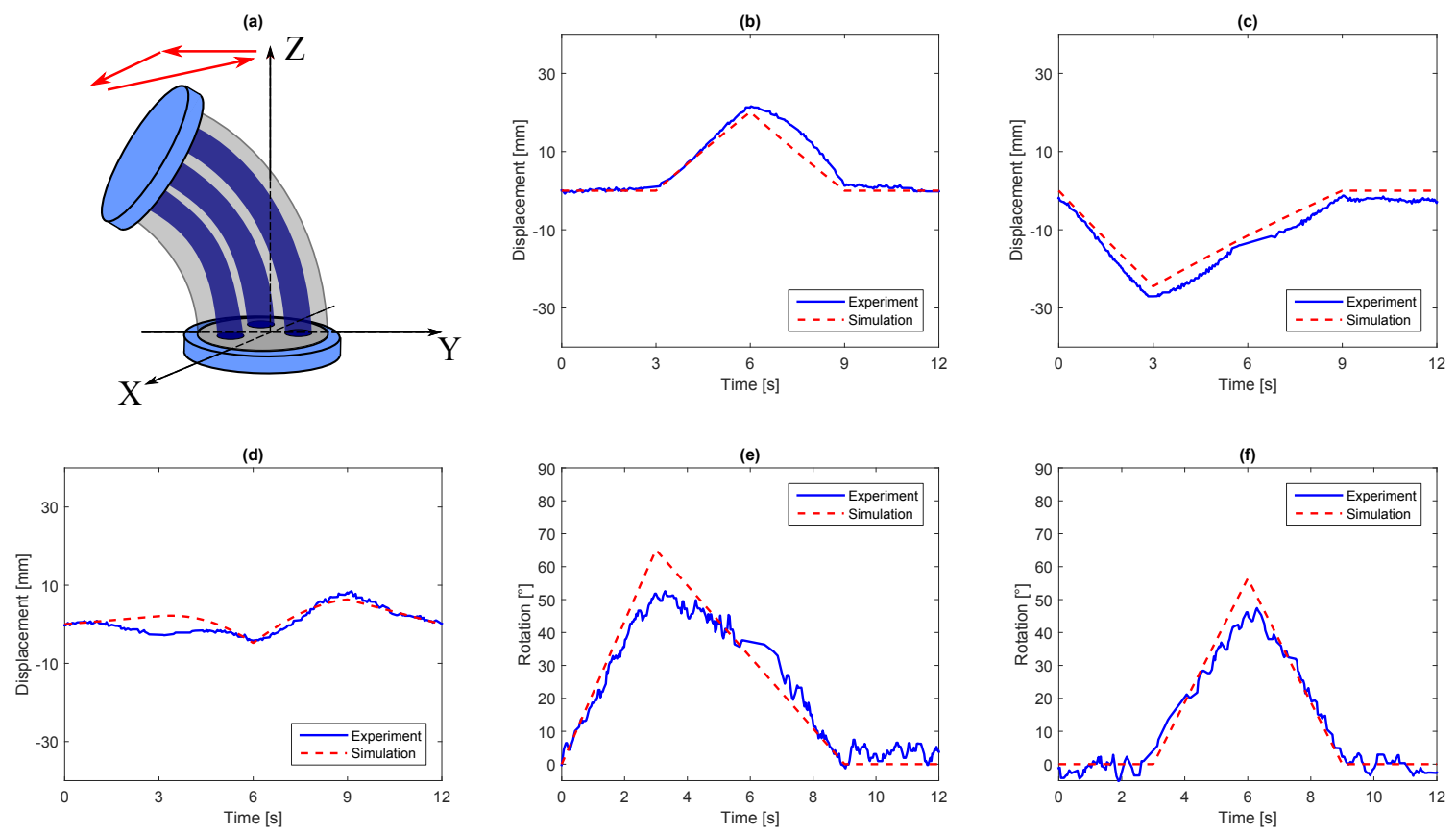

Fig. 5. Experimental results for second trajectory with (a) Trajectory visualization, deflection in $x-$ (b), y- (c) and z-axis (d) as well as rotation around $\mathrm{x}-(\mathrm{e})$ and $\mathrm{y}$-axis (f)

which is shown in figure 5(a), comprises a sequential motion beginning with an idle, deflated configuration. Chamber one is inflated from $0 \mathrm{kPa}$ to $80 \mathrm{kPa}$, followed by an inflation of chamber two and chamber three in the same pressure range before the three chambers are deflated simultaneously. For trajectories are evaluated in unloaded states. The deflections and rotations during the trajectory executions are presented in figures 4 and 5 . The maximum errors in position and orientation are summarized in table I.

\section{A. First trajectory}

The validation results of the first trajectory are presented in figure 4. It can be seen that for the displacement in $\mathrm{x}$-direction and the rotation about $\mathrm{y}$-axis both model experiment coincide at $0 \mathrm{~mm}$ and $0^{\circ}$ respectively. For the deflection in $\mathrm{y}$ - and $\mathrm{z}$-axis and the rotation about $\mathrm{x}$-axis deviations can be observed particularly at the point of 
TABLE I

MAXIMUM ERROR IN POSITION AND ORIENTATION

\begin{tabular}{ccc}
\hline Direction & Trajectory 1 & Trajectory 2 \\
\hline$\delta_{x}$ & $0 \mathrm{~mm}$ & $6 \mathrm{~mm}$ \\
$\delta_{y}$ & $6 \mathrm{~mm}$ & $5 \mathrm{~mm}$ \\
$\delta_{z}$ & $4 \mathrm{~mm}$ & $6 \mathrm{~mm}$ \\
$\theta_{x}$ & $18^{\circ}$ & $15^{\circ}$ \\
$\theta_{y}$ & $2^{\circ}$ & $10^{\circ}$ \\
\hline
\end{tabular}

maximum inflation $(6 \mathrm{~s})$, with maximum errors of $6 \mathrm{~mm}$, $4 \mathrm{~mm}$ and $18^{\circ}$ respectively. The latter could be explained by linearization of the modelling, which becomes less accurate for larger deflections. The deflection of the y-axis also shows a continuing error during the deflation phase (6s to $12 \mathrm{~s}$ ) which could occur due to hysteresis in the silicone rubber of the manipulator body.

\section{B. Second trajectory}

The experimental results of the second trajectory are shown in figure 5. For the deflections in $\mathrm{x}$ - and $\mathrm{y}$-axis it can be seen that the simulated and real trajectories coincide with maximum errors of $6 \mathrm{~mm}$ and $5 \mathrm{~mm}$. The experimental trajectory reveals non-linear behaviour during the inflation of chambers 2 and 3 ( $3 \mathrm{~s}$ to $9 \mathrm{~s}$ ). The occurrence of nonlinearities could be explained by the simultaneous inflation of multiple chambers, which might result in an amplification of the non-linear material effects. The deflection in $\mathrm{z}$ shows deviations with a maximum error of $6 \mathrm{~mm}$ in the first half of the trajectory ( $0 \mathrm{~s}$ to $6 \mathrm{~s})$ and matches closely for the second half. For the rotations about $\mathrm{x}$ - and $\mathrm{y}$-axis it can be observed that a maximum error occurs at the maximum deflection angle $(3 \mathrm{~s}$ for the rotation about $\mathrm{x}$ - and $6 \mathrm{~s}$ for the rotation about the y-axis respectively). The maximum errors are $15^{\circ}$ for the rotation about $\mathrm{x}$ - and $10^{\circ}$ for the rotation about the y-axis. The latter could be, similarly to the first trajectory, caused by the large deflection angle.

\section{CONCLUSION}

A methodology is presented to derive analytically kinematics equations and effects of external forces and moments of a soft robotics manipulator according to Timoshenko beam theory. The experimental validation of the method shows good accordance between model and physical manipulator for most parts of the verified trajectories. Deviations between the two can be observed particularly upon large deflections of the manipulator tip. The limitation could be overcome by introducing an adaptation of the model for large deflections. The method shows great potential to be universally applied to beam-like serial or parallel soft robotics manipulators based on a stiffness matrix derivation which is highly applicable to multi-segment soft robots. Furthermore, the method could be beneficial for real-time applications as the computational work to solve the kinematic equations is minimal. Future work involves model improvements for large deflections, the derivation of inverse kinematics and as well as static force applications.

\section{REFERENCES}

[1] D. Rus and M. T. Tolley, "Design, fabrication and control of soft robots," Nature, vol. 521, no. 7553, pp. 467-475, 2015.

[2] D. Trivedi, C. D. Rahn, W. M. Kier, and I. D. Walker, "Soft robotics: Biological inspiration, state of the art, and future research," Applied Bionics and Biomechanics, vol. 5, no. 3, pp. 99-117, Dec. 2008. [Online]. Available: http://www.tandfonline.com/doi/abs/10.1080/11762320802557865

[3] M. W. Hannan and I. D. Walker, "Kinematics and the implementation of an elephant's trunk manipulator and other continuum style robots." Journal of robotic systems, vol. 20, pp. 45-63, 2003.

[4] B. a. Jones, W. McMahan, and I. D. Walker, "Practical kinematics for real-time implementation of continuum robots," Proceedings - IEEE International Conference on Robotics and Automation, vol. 2006, no. 6 , pp. $1840-1847,2006$

[5] G. Chirikjian, "A continuum approach to hyper-redundant manipulator dynamics," Proceedings of 1993 IEEE/RSJ International Conference on Intelligent Robots and Systems (IROS '93), vol. 2, 1993.

[6] G. S. Chirikjian and J. W. Burdick, "The Kinematics of HyperRedundant Robot Locomotion," vol. 11, no. 6, 1995.

[7] R. J. Webster and B. a. Jones, "Design and Kinematic Modeling of Constant Curvature Continuum Robots: A Review," The International Journal of Robotics Research, vol. 29, no. 13, pp. 1661-1683, 2010.

[8] D. Trivedi, A. Lotfi, and C. D. Rahn, "Geometrically exact dynamic models for soft robotic manipulators," in IEEE International Conference on Intelligent Robots and Systems, vol. 24, no. 4, 2007, pp. 1497-1502.

[9] M. Cianchetti, T. Ranzani, G. Gerboni, T. Nanayakkara, K. Althoefer, P. Dasgupta, and A. Menciassi, "Soft Robotics Technologies to Address Shortcomings in Today's Minimally Invasive Surgery: The STIFF-FLOP Approach," Soft Robotics, vol. 1, no. 2, pp. 122-131, 2014.

[10] M. S. Malekzadeh, S. Calinon, D. Bruno, and D. G. Caldwell, "Learning by imitation with the STIFF-FLOP surgical robot : a biomimetic approach inspired by octopus movements," pp. 1-15, 2014.

[11] A. D. Marchese, K. Komorowski, C. D. Onal, and D. Rus, "Design and Control of a Soft and Continuously Deformable 2D Robotic Manipulation System," Proceedings of IEEE International Conference on Robotics and Automation. 2014, pp. 2189-2196, May 2014. [Online]. Available: http://ieeexplore.ieee.org/lpdocs/epic03/wrapper.htm?arnumber=690716

[12] H. Wang, W. Chen, X. Yu, T. Deng, X. Wang, and R. Pfeifer, "Visual servo control of cable-driven soft robotic manipulator," IEEE International Conference on Intelligent Robots and Systems, pp. 5762,2013

[13] T. Li, K. Nakajima, M. Calisti, C. Laschi, and R. Pfeifer, "Octopusinspired sensorimotor control of a multi-arm soft robot," 2012 IEEE International Conference on Mechatronics and Automation, ICMA 2012, pp. 948-955, 2012.

[14] T. Li, K. Nakajima, M. Kuba, T. Gutnick, B. Hochner, and R. Pfeifer, "From the octopus to soft robot control: an octopus inspired behaviour control architecture for soft robots," vol. 61, no. 4, pp. 211-217, 2012.

[15] J. Reddy, C. Wang, and K. Lee, "Relationships between bending solutions of classical and shear deformation beam theories," International Journal of Solids and Structures, vol. 34, no. 26, pp. 3373-3384, Sep. 1997.

[16] J. S. Przemieniecki, Theory of matrix structural analysis. Courier Corporation, 1985 